\title{
ANÁLISE ERGONÔMICA E POSTURAL DOS CITOLOGISTAS DE UM HOSPITAL NA CIDADE DE FORTALEZA (CE)
}

Thiago Brasileiro de Vasconcelos

Universidade Federal do Ceará (UFC), Fortaleza (CE), Brasil.

Ana Richelly Nunes Rocha Cardoso

Hospital Universitário Walter Cantídio; Universidade Federal do Ceará (UFC), Fortaleza (CE), Brasil.

Flávia Ravany Carneiro

Marklana da Frota Diniz

Cláudia Maria Montenegro

Andréa da Nóbrega Cirino Nogueira Vasco Pinheiro Diógenes Bastos

Centro Universitário Estácio do Ceará, Fortaleza (CE), Brasil.
RESUMO: O objetivo deste estudo foi analisar os aspectos ergonômicos e posturais dos citologistas, bem como a identificação das suas condições ergonômicas no ambiente de trabalho e os fatores que causam ou agravam as lesões por esforços repetitivos. Tratou-se de um estudo de caráter descritivo, observacional e transversal com estratégia de análise quantitativa dos resultados, realizado em um hospital localizado na cidade de Fortaleza (CE). A coleta de dados foi realizada de fevereiro a maio de 2013, através da aplicação de um questionário estruturado sobre assuntos ergonômicos e biomecânicos, e em seguida foi avaliada a condição ergonômica e biomecânica do posto de trabalho e a postura dos citologistas. Foram analisados 15 citologistas de ambos os sexos, $87 \%(\mathrm{n}=13)$ do sexo feminino e $13 \%$ $(\mathrm{n}=2)$ do sexo masculino, com média de tempo de trabalho de 27,2 $\pm 1,89$ anos e de tempo de trabalho na função atual de $18,73 \pm 1,77$ anos. Em relação à avaliação ergonômica e biomecânica, observamos que todos os citologistas $100 \%(n=15)$ apresentam repetitividade em sua jornada de trabalho e utilizam posições forçadas. A amostra pesquisada apresentou condições ergonômicas e biomecânicas razoáveis. Evidenciou-se a necessidade de mudanças no posto de trabalho com o intuito de melhorar a qualidade de vida, produtividade e postura dos citologistas, além de incentivar as pausas programadas e a adesão dos funcionários ao programa de Cinesioterapia Laboral.

PALAVRAS-CHAVE: Ergonomia; Modalidades de Fisioterapia; Postura.

\section{ERGONOMIC AND POSTURE ANALYSIS OF CYTOLOGISTS OF A HOSPITAL IN FORTALEZA, BRAZIL}

ABSTRACT: Current study analyzes the ergonomic and posture aspects of cytologists, ergonomic conditions in their work milieu and the factors that cause or worsen lesions by repeated activities. The descriptive, observational and transversal analysis features strategies for the quantitative investigation of results in a hospital in Fortaleza CE Brazil. Data were collected between February and May 2013 by applying a structured questionnaire on ergonomic and biomechanical items, coupled to the evaluation of the ergonomic and biomechanical condition of the labor milieu and the posture of the cytologists concerned. Fifteen cytologists, $87 \%(n=13)$ females and $13 \%(n=2)$ males, were analyzed, with average labor period of $27.2 \pm 1.89$ years and labor period as cytologists of $18.73 \pm 1.77$ years. In the ergonomic and biomechanical assessment, all cytologists $(n=15)$ practiced repetitions during their work session and employed forced positions. Sample showed mild ergonomic and biomechanical conditions and demonstrated the need of change in the milieu to improve life quality, productivity and posture, adopt programed pause and adhesion to the program for Work Kinesiotherapy.

KEY WORDS: Ergonomy; Posture; Modalities of Physiotherapy. 


\section{INTRODUÇÃO}

A Citologia ou Biologia Celular é o ramo da biologia que estuda as células no que diz respeito à estrutura, funções e sua importância na complexidade dos seres vivos. Com a invenção do microscópio óptico foi possível observar estruturas nunca antes vistas pelo homem, as células. Essas estruturas foram mais bem estudadas com o emprego de técnicas de citoquímica e o auxílio do microscópio eletrônico (ANDRADE, 2012; GRUPO IPED, 2012).

As atividades dos citologistas exigem que o trabalho seja executado na posição sentada durante praticamente toda a jornada de trabalho (SBC, 2012). Este fato pode vir a provocar tensionamentos, dores musculares e cansaço físico.

Segundo Pereira (2001) os esforços repetitivos, trabalho estático, ritmos intensos de trabalho, péssima condição biomecânica do posto de trabalho e posturas inadequadas estão presentes na maioria das atividades profissionais. Essas condições de trabalho são causas para o aparecimento ou agravamento de lesões, principalmente no sistema musculoesquelético.

Os termos Lesões por Esforços Repetitivos (LER) e Distúrbios Osteo-musculares Relacionados ao Trabalho (DORT) são usados para determinar as afecções que podem lesionar tendões, sinóvias, músculos, nervos, fáscias e ligamentos, de forma isolada ou associada, com ou sem degeneração dos tecidos, atingindo principalmente membros superiores, região escapular e pescoço. Decorrente de uma origem ocupacional ela pode ser ocasionada de forma combinada ou não por uso repetido e forçado de grupos musculares e da manutenção de posturas inadequadas (MELO, 2003).

As aplicações das análises ergonômicas e posturais nos postos de trabalho são muito úteis para a solução de problemas estruturais, por exemplo, a queda de produtividade, afastamento por auxílio-doença e aumento dos acidentes de trabalho. A má postura pode ser corrigida por meio de treinamentos específicos com a finalidade de adoção de posturas mais seguras, saudáveis e confortáveis (FIEDLER et al., 2003).

A abordagem fisioterapêutica no trabalho está em ascensão principalmente pela descoberta da importância do investimento na área preventiva e no combate aos distúrbios osteomusculares. Anualmente, as altas taxas de acidentes e doenças registradas pelas estatísticas oficiais expõem os elevados gastos e prejuízos humanos, sociais e econômicos que custam muito para o país, considerando apenas os dados do trabalho formal (RIBEIRO, 2009).

Nessa perspectiva, surgiu o interesse em realizar uma análise dos aspectos ergonômicos e posturais dos citologistas, bem como a identificação das suas condições ergonômicas no ambiente de trabalho e os fatores que causam ou agravam as lesões por esforços repetitivos, que uma vez eliminados ou minimizados, darão lugar ao processo natural de recuperação do organismo e melhor rendimento ao trabalho, possibilitando benefícios para a empresa, tais como diminuição das despesas médicas, hospitalares, faltas ao trabalho, absenteísmo e aposentadorias precoces. Sendo assim, o presente estudo objetivou analisar a ergonomia e postura dos citologistas de um hospital na cidade de Fortaleza (CE).

\section{MATERIAL E MÉTODOS}

Este estudo é de caráter descritivo, observacional e transversal com estratégia de análise quantitativa dos resultados, realizado no Instituto de Prevenção do Câncer (IPC), localizado na cidade de Fortaleza (CE). O IPC foi escolhido por ser um centro de referência e o local com maior número de citologistas no Estado do Ceará. A coleta de dados foi realizada de fevereiro a maio de 2013 após aprovação do Comitê de Ética em Pesquisa da Universidade Federal Ceará (Protocolo no 125/12).

Foram considerados como sujeitos do estudo os citologistas que trabalham no IPC, que aceitaram participar da pesquisa, assinando espontaneamente o Termo de Consentimento Livre e Esclarecido. Foram incluídos no estudo homens e mulheres citologistas que trabalham no IPC independentes do estado civil, religião, raça, status sócio-econômico ou grau de escolaridade e que manifestaram o desejo em participar da pesquisa. Foram excluídos os citologistas que apresentaram deformidades ortopédicas e/ou amputações, por serem agravos que, por si só, influenciam na postura e nas 
condições biomecânicas.

Inicialmente foi realizada uma reunião com a Coordenação do Centro de Estudos Rocha Furtado responsável pelas pesquisas no IPC e com a coordenadora do serviço de Citologia para autorizarem a realização da pesquisa com os citologistas da instituição (Figura 1).

Posteriormente, foi entregue o Termo de Consentimento Livre e Esclarecido aos participantes para maiores esclarecimentos sobre a pesquisa. A partir da assinatura do termo, foi garantido o total sigilo das informações obtidas e preservação dos princípios éticos, sendo, em seguida, dado início à coleta de dados.

A coleta de dados foi iniciada com a aplicação de um questionário estruturado sobre assuntos ergonômicos e biomecânicos (tempo de trabalho na empresa, tempo na função atual, rotatividade no posto de trabalho, programa de LER/DORT, atividade física, dor no corpo, iluminação, aspectos sonoros, existência de pausas durante o trabalho), e em seguida foi avaliada por um pesquisador fisioterapeuta a condição ergonômica e biomecânica do posto de trabalho por meio de uma ficha (MOTTA, 2009, modificado). Na avaliação do posto de trabalho, utilizou-se uma câmera fotográfica digital Sony Cyber-Shot ${ }^{\circledR}$ DSC-W320 14.1 megapixels.

A avaliação postural (vista anterior, lateral e posterior) (MENEZES, 2008; MELISCKI; MONTEIRO; GIGLIO, 2011) foi realizada nos horários disponíveis de cada citologista em uma sala reservada e tranquila disponibilizada pelo IPC.

Ressalta-se que as avaliações foram realizadas de forma individualizada, por um único pesquisador apto para realizá-las, diante disso, para a Avaliação Anterior os voluntários foram instruídos a ficar de pé (ortostatismo) de frente para o avaliador, com braços ao longo do corpo e com olhar fixo no horizonte - foram demarcados pontos de identificação nos acrômios, espinhas ilíacas ânterosuperiores e joelhos, além da palpação das cristas ilíacas para observação de sua altura.

Na Posição Lateral, o voluntário foi instruído a ficar em pé de lado para o avaliador, com braços ao longo do corpo e com olhar fixo no horizonte - foram demarcados pontos de identificação no tubérculo maior do úmero, ápice das curvaturas cervical, torácica e lombar:
Para avaliação na Posição Posterior, o voluntário foi instruído a ficar em pé de costas para o avaliador, com braços ao longo do corpo e com olhar fixo no horizonte - foram demarcados pontos de identificação nos ângulos inferiores da escápula e espinhas ilíacas póstero-superiores.

Também foi utilizada a Fotopodografia, por meio da realização de uma pintura na planta dos pés dos citologistas com tinta antialérgica, para que fossem obtidas as impressões plantares em posição ortostática, sobre uma folha de papel ofício em branco.

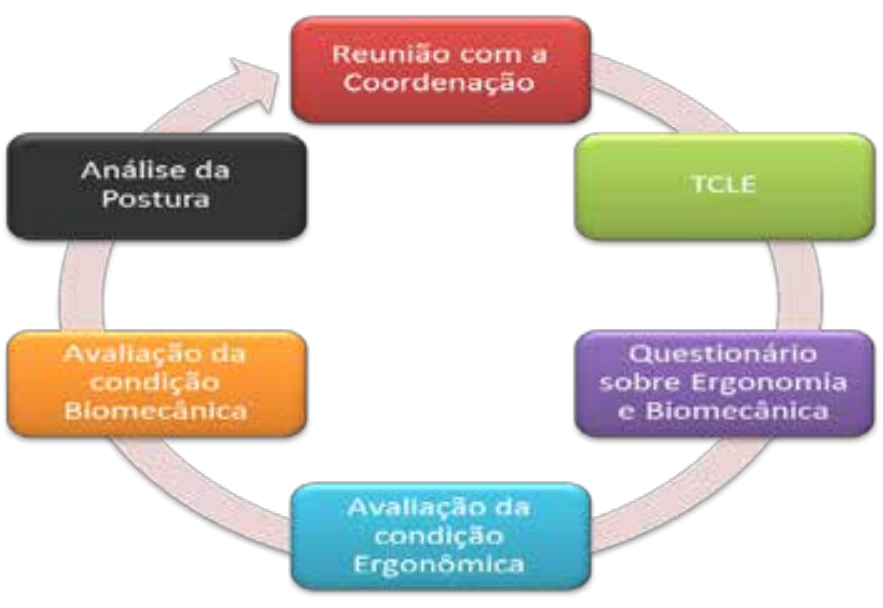

Figura 1. Diagrama do Processo de coleta de dados.

\section{RESULTADOS}

Nesse estudo foi analisada a ergonomia, biomecânica e postura de todos os 15 citologistas que atuam em um hospital localizado na cidade de Fortaleza (CE), sendo $87 \%(\mathrm{n}=13)$ do sexo feminino e $13 \%$ (n $=2$ ) do sexo masculino, com média de idade de 53,8 $\pm 2,19$ anos e Índice de Massa Corporal (IMC) de 25,67 $\pm 0,91$.

A média de tempo de trabalho foi de $27,2 \pm 1,89$ anos e de tempo de trabalho na função atual foi de 18,73 $\pm 1,77$ anos, sendo $73 \%(\mathrm{n}=11)$ citotécnicos e $27 \%(\mathrm{n}$ =4) citopatologistas. Dos profissionais pesquisados, $67 \%$ $(\mathrm{n}=10)$ informaram que nunca realizam rotatividade em sua jornada de trabalho, $53 \%(n=8)$ não participam do programa LER/DORT do hospital e $47 \%(\mathrm{n}=7)$ participam eventualmente do programa (Tabela 1).

Em relação à prática de exercício físico, $73 \%$ 
$(\mathrm{n}=11)$ informaram que não realizam, $20 \%(\mathrm{n}=3)$ afirmaram que praticam a caminhada e $7 \%(\mathrm{n}=1)$ a hidroginástica. Quando questionados sobre a sua opinião em relação ao programa de LER/DORT do hospital, $60 \%$ $(\mathrm{n}=9)$ não quiseram opinar (Tabela 1$)$.

Tabela 1. Distribuição dos dados relacionados ao trabalho dos citologistas e ao programa de LER/DORT

\begin{tabular}{lcc}
\hline Variáveis & N & f \% \\
\hline Tempo de trabalho & 2 & 13 \\
10 -0 20 anos & 10 & 67 \\
21 -0 30 anos & 3 & 20 \\
31 -0 40 anos & & \\
\hline Tempo na função atual & 9 & 60 \\
10 -0 20 anos & 6 & 40 \\
$210-030$ anos &
\end{tabular}

Tipo de tarefa

\begin{tabular}{lcc} 
Citotécnico & 11 & 73 \\
$\quad$ Citopatologista & 4 & 27 \\
\hline Rotatividade & & \\
$\quad$ Nunca & 10 & 67 \\
$\quad$ Eventualmente & 5 & 33 \\
\hline
\end{tabular}

\section{Participa do Programa LER/DORT}

Sim, eventualmente

Não participo

$7 \quad 47$

8

53

Opinião sobre o Programa de LER/ DORT

$\begin{array}{lll}\text { Observo melhora funcional } & 1 & 7 \\ \text { Bom, mas o horário é ruim } & 3 & 20 \\ \text { Não gostei } & 2 & 13 \\ \text { Sem opinião } & 9 & 60\end{array}$

Fonte: Elaborada pelos autores. Legenda: $\mathrm{N}=$ número de sujeitos; $\mathrm{f} \%=$ frequência relativa.

Em relação à avaliação ergonômica e biomecânica, observamos que todos os citologistas $100 \%$ $(\mathrm{n}=15)$ apresentam repetitividade em sua jornada de trabalho e utilizam posições forçadas, principalmente no tocante à flexão do antebraço. A amostra pesquisada apresentou na avaliação ergonômica uma média de 5,86 $\pm 0,23$ escores, sendo classificada como uma condição ergonômica razoável e na biomecânica uma média de 9 $\pm 0,19$ escores, sendo classificada como uma condição biomecânica razoável (Tabela 2).

Também foi questionado se os mesmos sentiam alguma dor no corpo, $67 \%(\mathrm{n}=10)$ citologistas afirmaram sentir dores localizadas na coluna lombar e nos membros superiores (antebraço e dedos). A intensidade da dor variou de 7,2 $\pm 0,52$, sendo classificada como moderada de acordo com a Escala Visual Analógica (EVA).

Tabela 2. Distribuição da amostra segundo a avaliação ergonômica e biomecânica do posto de trabalho

\begin{tabular}{|c|c|c|}
\hline Variáveis & $\mathbf{N}$ & f \% \\
\hline \multicolumn{3}{|c|}{ Posicionamento dos pés } \\
\hline Com apoio & 4 & 27 \\
\hline Sem apoio & 11 & 73 \\
\hline \multicolumn{3}{|l|}{ Largura da bancada } \\
\hline Suficiente & 1 & 7 \\
\hline Não suficiente & 14 & 93 \\
\hline \multicolumn{3}{|l|}{ Altura da bancada } \\
\hline Regulável & 0 & - \\
\hline Não regulável & 15 & 100 \\
\hline \multicolumn{3}{|l|}{ Angulação da cadeira } \\
\hline Ajustável & 0 & - \\
\hline Não ajustável & 15 & 100 \\
\hline \multicolumn{3}{|l|}{ Visão } \\
\hline Livre de reflexos & 11 & 73 \\
\hline Com reflexos & 4 & 27 \\
\hline \multicolumn{3}{|l|}{ Clima e som } \\
\hline Adequados & 15 & 100 \\
\hline Inadequados & 0 & - \\
\hline \multicolumn{3}{|c|}{ Avaliação ergonômica } \\
\hline Boa & 3 & 20 \\
\hline Razoável & 12 & 80 \\
\hline \multicolumn{3}{|l|}{ Alterna de posição } \\
\hline Sim & 2 & 13 \\
\hline Não & 13 & 87 \\
\hline
\end{tabular}

Pausas durante a jornada de trabalho

$\begin{array}{lcc}\text { Sim, } 1 \bigcirc-05 \text { minutos } & 2 & 13 \\ \text { Sim, } 6 \text {-o } 10 \text { minutos } & 2 & 13 \\ \text { Não } & 11 & 74\end{array}$

\begin{tabular}{lcc}
\hline Alterna de posição & & \\
Boa & 2 & 13 \\
Razoável & 13 & 87 \\
\hline
\end{tabular}

Fonte: Elaborada pelos autores. Legenda: $\mathrm{N}=$ número de sujeitos; $\mathrm{f} \%=$ frequência relativa.

Quando os citologistas foram questionados sobre sugestões para a melhoria do posto de trabalho, 
apenas $40 \%(n=6)$ opinaram, e os mesmos afirmaram que seria necessário melhorar o espaço da bancada ou cabine de trabalho, tanto na largura como na altura e utilizar cadeiras mais confortáveis e reguláveis na altura do encosto e na altura dos cotovelos (Figura 2).
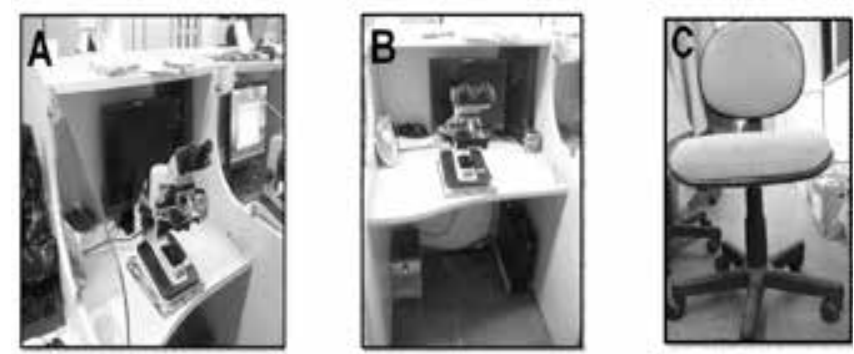

Figura 2. Imagens do posto de trabalho dos citologistas.

Legenda: A: Imagem ântero-superior da bancada de trabalho. B: Imagem anterior do posto

de trabalho dos citologistas. C: Imagem da cadeira utilizada pelos citologistas.

$\mathrm{Na}$ análise postural observamos alterações principalmente nas regiões cervical e torácica, como na inclinação da cabeça $87 \%(n=13)$, assimetria dos ombros $80 \%(\mathrm{n}=12)$, assimetria do Triângulo de Thales $100 \%$ ( $=15)$, hiperlordose lombar 53\% $(\mathrm{n}=8)$, presença de gibosidade $60 \%(\mathrm{n}=9)$ e assimetria das cristas ilíacas $80 \%(n=12)$ (Tabela 3).

Tabela 3. Distribuição percentual da amostra segundo a avaliação postural anterior, lateral e posterior

\begin{tabular}{lcc} 
& & (Continua) \\
\hline Variáveis & N & f \% \\
\hline Posição da cabeça & 2 & 13 \\
$\quad$ Centralizada & 7 & 47 \\
$\quad$ Lateralizada à D & 6 & 40 \\
$\quad$ Lateralizada à E & & \\
\hline Ombros & 3 & 20 \\
$\quad$ Normais & 6 & 40 \\
Direito elevado & 6 & 40 \\
$\quad$ Esquerdo elevado & & \\
\hline Proeminência Escapular & 5 & 33 \\
Simétricas & 7 & 47 \\
Abduzidas & 3 & 20 \\
Aduzidas & & \\
\hline Triângulo de Thales & & - \\
Simétricos & 0 & 47 \\
Menor à D & 7 & 53 \\
Menor à E & 8 &
\end{tabular}

\begin{tabular}{lcc}
\hline Variáveis & N & f \% \\
\hline Coluna lombar & & \\
$\quad$ Normal & 4 & 27 \\
$\quad$ Hiperlordose & 8 & 53 \\
$\quad$ Retificada & 3 & 20 \\
\hline Teste de Adams & & \\
$\quad$ Sem gibosidade & 6 & 40 \\
$\quad$ Gibosidade à D & 5 & 33 \\
$\quad$ Gibosidade à E & 4 & 27 \\
\hline Crista Ilíaca & & \\
Simétricas & 3 & 20 \\
Maior à D & 4 & 27 \\
$\quad$ Maior à E & 8 & 53 \\
\hline Joelhos & & \\
$\quad$ Neutros & 7 & 47 \\
$\quad$ Varo & 0 & - \\
$\quad$ Valgo & 8 & 53 \\
\hline Pés & & \\
Neutros & 9 & 60 \\
Cavos & 3 & 20 \\
Planos & 3 & 20 \\
\hline
\end{tabular}

Fonte: Elaborada pelos autores.

\section{DISCUSSÃO}

No presente estudo, foi evidenciado que os citologistas possuem idade elevada, vários anos de atividade profissional, são sedentários e estão acima do peso ideal, o que pode ocasionar algumas disfunções, por exemplo, problemas cardiovasculares, LERs e DORTs e alterações posturais. Carneiro et al. (2012) confirmam nossos dados, destacando que durante a fase produtiva, $o$ corpo humano sofre alterações decorrentes dos esforços aos quais é submetido. É importante destacar que, além destas alterações, todas as funções do corpo humano são fortemente influenciadas pelo processo degenerativo ligado aos vários aspectos biopsicossociais envolvidos.

Segundo Bienfait (2000) cada indivíduo adota uma postura diferente, que pode ser influenciada por fatores estruturais, o que significa dizer que ela pode ser alterada por causas anatômicas ou fatores posicionais, que estão relacionadas aos hábitos posturais adotados pelos indivíduos.

O programa Cinesioterapia Laboral (LER/DORT) 
está inserido no hospital pesquisado, entretanto, apenas $47 \%$ dos citologistas participam eventualmente do programa, e muitos destacaram que a maior dificuldade na adesão seria por conta do horário proposto. Carneiro et al. (2012) e Souza et al. (2012) afirmam que a Cinesioterapia Laboral pode trazer ótimos benefícios para a empresa, como o aumento da produtividade, menores gastos médicos, diminuição de doenças ocupacionais, e para 0 empregado, a redução de dores, aumento da autoestima, redução de estresse, melhoria na relação interpessoal, aumento da disposição, motivação e melhoria da saúde bio-psico-social.

Algumas medidas podem ser realizadas com $o$ intuito de motivar a participação dos funcionários no programa de prevenção de LER/DORT, por exemplo, propor um melhor horário de execução do programa, utilizar cartilhas ilustrativas, vídeos e aulas motivacionais sobre a importância de prevenir LER/DORT, dentre outros. Para Negrete e Lartigue (2004) a facilidade com que esses instrumentos comunicam conhecimentos científicos está relacionada ao fato de que eles transmitem informações de forma atrativa, divertida e facilita a memorização de conceitos.

Para Cardoso e Popolim (2006) a avaliação ergonômica destina-se a verificar e qualificar as condições a que estão sujeitos os funcionários em suas atividades durante o processo produtivo da empresa. A influência sobre a qualidade de vida do ser humano dentro da empresa é reflexo do ambiente de trabalho como um todo. A Avaliação Ergonômica está diretamente ligada à ergonomia de manutenção (corretiva) onde o trabalho é analisado conforme a tarefa que já é executada.

Observou-se que o posto de trabalho possui uma condição ergonômica e biomecânica razoável o que pode interferir na produtividade dos citologistas. Para Parada, Alexandre e Benatti (2002), o ambiente de trabalho, sob condições físicas, mecânicas e psíquicas adversas, é considerado como um dos principais fatores de risco para o desenvolvimento de alterações no sistema musculoesquelético. A exposição contínua e prolongada do corpo aos fatores de risco de tal ambiente favorece o surgimento das doenças ocupacionais (GURGUEIRA; ALEXANDRE; CORRÊA-FILHO, 2003).

Sendo assim, Bloemer (2001), Grandjean (2004) e Brasil (2006) destacam que uma adequação do posto de trabalho ao trabalhador oferece inúmeras vantagens, tais como: melhor qualidade de vida; diminuição da fadiga e desconforto físico; diminuição dos gastos com assistência médica; aumento da eficiência do trabalho; maior proteção legal à empresa; aumento da produtividade; melhor ambiente de trabalho; e favorecimento da socialização do trabalhador junto ao grupo de trabalho.

A postura sentada é a postura mais adotada pelos citologistas, pois passam quase que a totalidade da jornada de trabalho nessa posição, sem pausas nem rotatividade e com atividades repetitivas. Moraes (2002) e Lippert (2010) destacam que a posição sentada pode originar uma série de dores e complicações advindas da pressão exercida sobre os discos intervertebrais, já que nessa posição a pressão pode ser até $50 \%$ maior do que em bipedestação, acarretando maior carga à coluna vertebral. Além dos problemas lombares, a postura sentada prolongada tende a reduzir a circulação de retorno dos membros inferiores, gerando edema nos pés e tornozelos e, também, promove desconfortos na região do pescoço e membros superiores (COURY; RODGHER, 1997).

Braccialli e Vilarta (2000) acrescentam que o modelo biomecânico da coluna do homem não foi construído para permanecer por longos períodos na posição sentada, mantendo posturas estáticas fixadas e realizando movimentos repetitivos.

Os citologistas pesquisados informaram que seria necessário melhorar o espaço da cabine de trabalho e utilizar cadeiras mais confortáveis e reguláveis. Brandimiller (1999) corrobora com o presente estudo quando afirma que, para assegurar o conforto no trabalho, uma cadeira ergonômica é fundamental, porém será de pouca valia se a mesa não for adequada para o tipo de trabalho ou para suas dimensões corporais.

Por intermédio da análise ergonômica do trabalho é possível não somente categorizar as atividades dos trabalhadores, como também permitir modificar o ambiente do trabalho ao modificar a atividade exercida, buscando a adaptação do trabalho ao homem. A mesma atividade realizada por diferentes trabalhadores nem sempre é realizada segundo um único protocolo. Possivelmente em função da natureza da tarefa, das 
diferentes formas de executá-la e de interagir com a organização do trabalho, alguns indivíduos apresentam problemas de saúde que podem se manifestar de diferentes formas, como as DORTs (VICENTINE, 2012).

Quando questionados sobre a presença de dor no corpo, a grande maioria (67\%) afirmou sentir dores localizadas principalmente na coluna lombar e nos membros superiores (antebraço e dedos), já que são as áreas mais sobrecarregadas durante sua atividade laboral, e com intensidade elevada, sendo classificada como dor moderada. Walsh et al. (2004) e Souza et al. (2012) afirmam que é elevada a frequência de dores lombares em pessoas que executam o trabalho, tanto na posição sentada como em pé. A frequência destes distúrbios leva a suspeitar de uma não correta adaptação da máquina ao homem, bem como de posturas incorretas dos trabalhadores.

Zapater et al. (2004) acrescentam que o planejamento e/ou replanejamento do ambiente físico, através da adoção de mobiliário ajustável a diferentes requisitos da tarefa e às medidas antropométricas individuais, é uma forma de minimizar os efeitos adversos da postura sentada para as estruturas músculoesqueléticas, porém, apenas esta forma de intervenção não é suficiente para minimizar o problema.

Os citologistas pesquisados apresentaram disfunções posturais, mais acentuadas nas regiões cervical e torácica, o que pode estar relacionado com o posto de trabalho, pois são regiões utilizadas diariamente durante a jornada de trabalho. Corroborando com nossos dados, Moraes (2002), Magee (2002) e Dul e Weerdmeester (2004) afirmam que hábitos posturais inadequados, como os impostos pela posição sentada, agindo sobre o organismo humano de forma repetitiva são capazes de levar seus vários mecanismos de defesa a ações compensatórias.

Moares (2002), Lida (2005) e Souza et al. (2012) acrescentam que indivíduos que adotam frequentemente uma mesma posição corporal durante a jornada de trabalho podem gerar alterações significativas no alinhamento corporal, além de apresentarem dor ou desconforto na musculatura mais utilizada. $\mathrm{O}$ indivíduo acaba adquirindo vícios posturais, além de outros problemas oriundos do trabalho. Estes, se não forem prevenidos ou amenizados com informações sobre seu posto de trabalho e os riscos que o envolvem, a médio e longo prazo afetarão seu desempenho no cotidiano e na saúde e, por consequência, sua qualidade de vida.

No estudo de Souza et al. (2012), que avaliou a dor e lesões ocasionadas pelo trabalho em cirurgiõesdentistas, os autores destacaram que é elevada a prevalência de LER/DORT nos profissionais pesquisados, e destacam a necessidade de uma atuação preventiva e corretiva do mobiliário com o objetivo de minimizar os distúrbios musculoesqueléticos e proporcionar mais qualidade de vida aos funcionários.

Grandjean (2004) acrescenta afirmando que é de extrema importância a adoção de um programa de orientação postural para atividades laborais e, ao mesmo tempo, inserir outras atividades como a diminuição da repetitividade e da jornada de trabalho, com o intuito de reduzir os esforços musculares do trabalho estático.

As limitações deste estudo estão relacionadas ao pequeno tamanho amostral e pela pesquisa ter sido realizada em apenas um posto de trabalho dos citologistas, entretanto, vale ressaltar que o hospital pesquisado é um centro de referência em citologia no Estado do Ceará, com elevada solicitação de exames citopatológicos e o local com maior número de citologistas. Recomendamos novos estudos, de modo a permitir delinear o perfil epidemiológico e ergonômico-postural desse importante ramo de atividade profissional.

\section{CONCLUSÃO}

Os citologistas pesquisados são em sua maioria do sexo feminino, possuem média de idade e de tempo de trabalho elevados, são sedentários, estão acima do seu peso ideal e dificilmente realizam rotatividade em sua jornada de trabalho.

Em relação ao posto de trabalho pesquisado foi observado que possui visão livre de reflexos, clima e sons adequados o que beneficia a concentração.

Destacamos algumas medidas interessantes para uma maior adesão ao programa de prevenção de LER/DORT, dentre elas, adequação do horário, uso de cartilhas educativas, aulas explicativas sobre LER/DORT e vídeos. 
Evidenciou-se a necessidade de mudanças no posto de trabalho com o intuito de melhorar a qualidade de vida, produtividade e postura dos citologistas. As mudanças destacadas pelos profissionais, e comprovadas pelos pesquisadores, devem ser realizadas na largura e altura da mesa de trabalho, além da aquisição de cadeiras mais confortáveis que possuam regulagem da altura do encosto e da altura dos cotovelos, pausas programadas e aumento na adesão de funcionários ao programa de LER/ DORT.

Inferimos que o posto de trabalho não está adequado para a atividade laboral dos citologistas, sendo necessárias modificações ergonômicas com o intuito de melhorar a biomecânica e postura dos trabalhadores.

\section{REFERÊNCIAS}

ANDRADE, O. O que é citologia? Disponível em: < http:// mundonanet.sites.uol.com.br/biologia11.html > . Acesso em: 21 jan. 2012.

BIENFAIT, M. As bases da fisiologia da terapia manual. São Paulo: Summus, 2000.

BLOEMER, R. Postura e desconforto corporal em um ambiente de trabalho informatizado. 2001. $14 \mathrm{f}$. (Monografia) - Universidade do Sul de Santa Catarina UNISUL. Tubarão.

BRACCIALLI, L. M. P.; VILARTA, R. Aspectos a serem considerados na elaboração de programas de prevenção e orientação de problemas posturais. Rev Paulista Educ Física, São Paulo, v. 14, n. 2, p. 159-71, 2000.

BRANDIMILLER, P. A. O corpo no trabalho: guia de conforto e saúde para quem trabalha em microcomputadores. São Paulo: SENAC, 1999.

BRASIL. Ministério da Saúde. Secretaria de Atenção à Saúde. Departamento de Ações Programáticas Estratégicas. Lesões por Esforços Repetitivos (LER), Distúrbios Osteomusculares Relacionados ao Trabalho (DORT), dor relacionada ao trabalho: protocolos de atenção integral à saúde do trabalhador de complexidade diferenciada. Brasília: Ministério da Saúde, 2006.
CARDOSO, D. D.; POPOLIM, F. D. Alterações da coluna vertebral relacionadas ao trabalho sentado em costureiras. 2006. 37 f. Monografia (Graduação em Fisioterapia) - Centro Universitário Claretiano. Batatais, SP.

CARNEIRO, I. P.; CARNEIRO NETO, J. A.; ANDRADE, E. A.; NOGUEIRA, A. N. C.; CÂMARA, T. M. S.; NOGUEIRA, M. M. et al. Programa de cinesioterapia laboral para trabalhadores administrativos da empresa Companhia de Gestão dos Recursos Hídricos. Rev Fisioter \& Saúde Funcional, Fortaleza, v. 1, n. 1, p. 10-5, 2012.

COURY, H. J. C.; RODGHER, S. Treinamento para o controle de disfunções músculo-esqueléticas ocupacionais: um instrumento eficaz para a fisioterapia preventiva. Rev Bras Fisioter, São Carlos, v. 2, n. 1, p. 7-17, 1997.

DUL, J.; WEERDMEESTER, B. Ergonomia prática. 2. ed. São Paulo: Edgar Blucher, 2004.

FIEDLER, N. C.; MENEZES, N. S. M.; AZEVEDO, I. N. C.; SILVA, J. R. M. Avaliação biomecânica dos trabalhadores em marcenarias no Distrito Federal. Ciênc Florest, Santa Maria, v. 13, n. 2, p. 99-109, 2003.

GRANDJEAN, E. Manual de ergonomia: adaptando o trabalho ao homem. 4. ed. Porto Alegre: Artes Médicas, 2004.

GRUPO IPED. Colégio Web. Citologia. Disponível em: $<$ http:www.colegioweb.com.br>. Acesso em: 07 set. 2012.

GURGUeIRA, G. P.; ALEXANDRE, N. M. C.; CORRÊAFILHO, H. R. Prevalência de sintomas musculoesqueléticos em trabalhadores de enfermagem. Rev Latino-am Enfermagem, Ribeirão Preto, v. 11, n. 5, p. 608-13, 2003.

LIDA, I. Ergonomia: projeto e produção. 2. ed. São Paulo: Edgard Blucher, 2005.

LIPPERT, L. S. Cinesiologia clínica e anatomia. 4. ed. Rio de Janeiro: Guanabara Koogan, 2010.

MAGEE, D. Avaliação Musculoesquelética. São Paulo: Manole, 2002. 
MELISCKI, G. A.; MONTEIRO, L. Z.; GIGLIO, C. A. Avaliação postural de nadadores e sua relação com o tipo de respiração. Fisioter Mov, Curitiba, v. 24, n. 4, p. 72128, 2011.

MELO, C. D. Doenças ocupacionais com ênfase a LER/ DORT. 2003. 70 f. Monografia (Especialização em Gestão Universitária) - Universidade Federal de Santa Catarina. Florianópolis, SC.

MENEZES, T.V.Estudocomparativodocomportamento postural de bailarinas da dança clássica. 2008. 55 f. Monografia (Fisioterapia) - Faculdade Integrada do Ceará. Fortaleza, CE.

MORAES, L. F. S. Os princípios das cadeias musculares na avaliação dos desconfortos corporais e constrangimentos posturais em motoristas do transporte coletivo. 2002. 133 f. Dissertação. (Engenharia de Produção) - Universidade Federal de Santa Catarina. Florianópolis, SC.

MOTTA, F. V. Avaliação ergonômica de postos de trabalho no setor de pré-impressão de uma indústria gráfica. 2009. $50 \mathrm{f}$. Trabalho de conclusão de curso (Engenharia de Produção) - Universidade Federal de Juiz de Fora, Juiz de Fora, MG.

NEGRETE, A.; LARTIGUE, C. Learning from education to communicate science as a good story. Endeavour, v. 28, n. 3, p. 120-24, 2004.

PARADA, E. O.; ALEXANDRE, N. M. C.; BENATTI, M. C. C. Lesões ocupacionais afetando a coluna vertebral em trabalhadores de enfermagem. Rev Latino-am Enfermagem, Ribeirão Preto, v. 10, n. 1, p. 64-9, 2002.

PEREIRA, E. R. Fundamentos de ergonomia e fisioterapia do trabalho. 2. ed. Rio de Janeiro: Taba Cultura, 2001.

RIBEIRO, E. P. Análise postural verificada através da biofotogametria após uso do SEATBALL em cirurgióes dentistas do Cais Nova Era. 2009. 68 f. Monografia Graduação (Fisioterapia) - Universidade Estadual de Goiás, Escola Superior de Educação Física e Fisioterapia do Estado de Goiás, Goiânia, GO.

SBC. Sociedade Brasileira de Citopatologia. História.
Disponível em: < http://portalsbc.com.br/historia.asp> Acesso em: 06 jan. 2012.

SOUZA, I. M. A.; VASCONCELOS, T. B.; BASTOS, V. P. D.; FARIAS, M. S. Q. Avaliação da dor e lesões ocasionadas pelo trabalho em cirurgióes-dentistas na cidade de Fortaleza/CE. Rev Fisioter \& Saúd Funcional, Fortaleza, v. 1, n. 2, p. 35-41, 2012.

VICENTINE, E. R. N. Lesões por esforços repetitivos x construção civil. Disponível em: <http://www. dornascostas.com.br/publicacoes.htm $>$. Acesso em: 26 nov. 2012.

WALSH, D. A.; KELLY, J. S.; JOHNSON, S. P.; RAJKUMAR, S.; BENNETTS, K. Performance problems of patients with chronic low-back pain and the measurement of patientcentered outcome. Spine, Hagerstown, v. 29, n. 1, p. 87 93, 2004 .

ZAPATER, A. R.; SILVEIRA, D. M.; VITTA, A.; PADOVANI, C. R.; SILVA, J. C. P. Postura sentada: a eficácia de um programa de educação para escolares. Ciênc saúde coletiva, Rio de Janeiro, v. 9, n. 1, p. 191-99, 2004.

Recebido em: 31 de maio de 2016 Versão final recebida em: 16 de agosto de 2016 Aceito em: 22 de agosto de 2016 